\title{
Evaluación de un taller práctico sobre higiene de manos impartido por estudiantes entrenados
}

\author{
María Fernández-Prada, Joaquín González-Cabrera, Yolanda Ortega-López, María Martínez-Bellón, \\ Milagros Fernández-Crehuet, Aurora Bueno-Cavanillas
}

Introducción. La enseñanza y el aprendizaje de la higiene de manos en el contexto sanitario es una tarea compleja. La intervención del estudiante como líder de su propia formación está muy poco analizada en la bibliografía. El objetivo principal del trabajo es la evaluación de un taller sobre higiene de manos gestionado, dirigido e impartido, bajo tutela experta, por estudiantes al propio colectivo estudiantil.

Sujetos y métodos. Evaluación pre-post de la técnica, los conocimientos y las actitudes de los participantes hacia la higiene de manos. Asistieron 40 estudiantes de ciencias de la salud de la Universidad de Granada. Se realizaron dos evaluaciones del taller: mediante un cuestionario diseñado ad hoc con 12 ítems sobre conocimientos y actitudes, y mediante la observación directa de la técnica de higiene de manos y la calidad del proceso utilizando una lámpara de luz ultravioleta y una solución reactiva.

Resultados. Tras la realización del taller se aprecia que disminuye de forma significativa el número de zonas contaminadas de las manos $(t=9,278 ; p<0,000)$ y que mejora significativamente la técnica de higiene de manos para cada uno de los siete pasos establecidos por la Organización Mundial de la Salud $(p<0,001)$. La diferencia entre la media global de las puntuaciones del cuestionario antes y después del taller fue también significativa $(t=-4,662 ; p<0,000)$.

Conclusiones. El taller impartido mejoró las actitudes, los conocimientos y la técnica de higiene de manos con solución alcohólica entre los participantes.

Palabras clave. Educación sanitaria. Higiene de manos. Liderazgo estudiantil.

\section{Evaluation of a workshop on hand hygiene taught by instructed students}

Introduction. The teaching and learning of hand hygiene in the context of health is a complex task. There is little discussion in the literature regarding the student involvement as the leader of his own background. The main objective of this study is the evaluation of a workshop on hand hygiene managed, directed and delivered, under expert supervision, by students at the same student group.

Subjects and methods. An evaluation pre-post about technique, knowledge and attitudes of participants towards hygiene. Attended by 40 students of Health Sciences at the University of Granada. We conducted two evaluations of the workshop by: a questionnaire designed ad hoc with 12 items on knowledge and attitudes, and the direct observation of hand hygiene technique and quality of the processing with an ultraviolet lamp and a reactive solution for it.

Results. After the workshop, it shows how significantly reduces the number of contaminated areas hands $(t=9.278$; $p<0.000$ ) and how it significantly improves hand hygiene technique for each of the seven steps outlined World Health Organization $(p<0.001)$. The difference between the overall mean questionnaire scores before and after the workshop was also significant $(t=-4.662 ; p<0.000)$.

Conclusions. The taught workshop improved attitudes, knowledge and technique of hand hygiene with alcohol solution between the participants.

Key words. Hand hygiene. Health education. Student leadership.
Servicio de Medicina Preventiva; Hospital Universitario San Cecilio; Granada (M. Fernández-Prada, M. Martínez-Bellón, A. BuenoCavanillas). Departamento de Psicología Social y Metodología de las Ciencias del Comportamiento (J. González-Cabrera); Departamento de Medicina Preventiva y Salud Pública (M. Fernández-Crehuet, A. Bueno-Cavanillas); Universidad de Granada. Servicio de Medicina Preventiva; Hospital Infanta Margarita; Cabra, Córdoba (Y. Ortega-López). CIBER de Epidemiología y Salud Pública, CIBERESP (A. Bueno-Cavanillas).

Correspondencia:

Dra. María Fernández Prada. Servicio de Medicina Preventiva. Hospital Universitario San Cecilio Avda. Doctor Olóriz, 16. E-18012 Granada.

Fax:

+34958246118.

E-mail:

mfdezprada@ugr.es

Agradecimientos:

A los miembros de la Asociación de Estudiantes Ramón y Cajal.

Conflicto de intereses:

No declarado.

Conflict of interests: None declared.

C 2012 Educación Médica 


\section{Introducción}

Una higiene de manos correcta y oportuna es la medida más importante para el control de las infecciones asociadas a la asistencia sanitaria. La formación en higiene de manos es la estrategia más utilizada para mejorar el porcentaje de cumplimiento de las indicaciones de higiene de manos [1]. Sin embargo, las intervenciones destinadas a aumentar el cumplimiento de las recomendaciones de higiene de manos tienen un efecto limitado que se diluye en el tiempo [2,3] y el cumplimiento global, salvo excepciones, sigue siendo inferior a lo deseado.

Las razones que alegan los profesionales para explicar su bajo cumplimiento pueden actuar como refuerzos negativos (falta de tiempo, agresión de los productos, rutina), mientras que los refuerzos positivos son escasos [4]. Entre las razones últimas, debe admitirse que los profesionales sanitarios han automatizado una serie de pautas de actuación muy difíciles de modificar $[4,5]$. Así pues, esta situación tiende a perpetuarse en el tiempo, ya que el aprendizaje de la higiene de manos es de tipo vicario fundamentalmente y tiene lugar durante las prácticas asistenciales en las que el estudiante aprende las conductas que observa, asumiéndolas en gran medida como correctas [4].

Inculcar activamente normas para la higiene de manos en los profesionales en formación tendría un doble beneficio. En primer lugar, éstos no han adquirido hábitos erróneos en la práctica asistencial, es más factible llegar a todos y son más receptivos al proceso de enseñanza-aprendizaje [4,5]. En segundo lugar, los estudiantes, observadores críticos, podrían servir como un refuerzo positivo de la conducta de los profesionales en relación con la higiene de manos si se les alienta para ello, tal como ha quedado de manifiesto en el trabajo de Henderson et al [6] sobre la implantación de la lista de verificación quirúrgica [7]. Si los complementos formativos son liderados por el propio colectivo, las probabilidades de éxito pueden ser mayores [7]. Igualmente, como señalan los propios estudiantes, diversos proyectos han podido desarrollarse gracias a la estrecha colaboración entre profesores, alumnos y otros miembros de sus facultades que han supuesto un apoyo crucial para la puesta en marcha de cursos formativos bajo demanda [8]. El objetivo principal de este trabajo es evaluar los resultados de un taller práctico sobre higiene de manos organizado y liderado por estudiantes y dirigido a ellos, bajo la tutela académica de personal experto.

\section{Sujetos y métodos}

\section{Diseño}

Estudio de evaluación pre-post de la técnica, los conocimientos y las actitudes de los participantes hacia la higiene de manos.

\section{Período y ámbito de estudio}

El taller se realizó en la Facultad de Medicina de la Universidad de Granada durante la primera semana del mes de abril de 2009, en dos días alternos (lunes y miércoles), con una duración de 10 horas en total.

\section{Participantes}

Participaron 40 estudiantes: 22 alumnos de la Facultad de Medicina y 18 de la Escuela Universitaria de Ciencias de la Salud (Enfermería y Fisioterapia). La edad media era de $21 \pm 2,65$ años y el porcentaje de mujeres, del 80,1\%.

\section{Desarrollo del taller}

El taller fue impartido por y para estudiantes de ciencias de la salud a través de la Asociación de Estudiantes de Medicina Ramón y Cajal. Fue ésta la que mostró interés en su realización, la que propuso y gestionó el taller, la que consiguió las fuentes de financiación y la que pidió colaboración y tutela. Por tanto, la idea nació en el seno estudiantil y fue apoyada por el Departamento de Medicina Preventiva y Salud Pública de la Universidad de Granada. Ocho fueron los estudiantes que tuvieron el papel de 'monitores'. Éstos habían sido participantes en una edición anterior del taller y, por consiguiente, eran conocedores de la teoría y de la técnica de higiene de manos. Con el fin de prepararlos para la realización del taller, recibieron una sesión específica de 90 minutos de duración por parte de personal del departamento. Esta sesión se centró en intentar que los estudiantes fueran dinamizadores sociales en cada uno de sus grupos y en explicarles el proceso de toma de muestras y recogida de datos.

Además, el departamento se encargó de supervisar las actividades académicas llevadas a cabo en todo momento. Los participantes se dividieron en grupos de trabajo liderados por los monitores.

El primer día, tras una charla introductoria de una hora de duración sobre la importancia de la higiene de manos en el ámbito hospitalario, impartida por una médico interno residente de cuarto año de Medicina Preventiva y Salud Pública, se realiza- 
Tabla I. Encuesta de opinión sobre higiene de manos.

1. ¿Cómo de importante consideras la higiene de manos en la práctica clínica?

2. ¿Con qué frecuencia crees que se transmiten infecciones en el hospital debido a la mala higiene de manos por parte del personal sanitario?

3. ¿Crees que es necesaria la formación en higiene de manos del personal sanitario?

4. En tu experiencia como estudiante, ¿qué frecuencia de cumplimiento crees que hay de las recomendaciones de higiene de manos por parte del personal de enfermería?

5. En tu experiencia como estudiante, ¿qué frecuencia de cumplimiento crees que hay de las recomendaciones de higiene de manos por parte del personal médico?

6. ¿Cómo de importante valoras la higiene de manos antes del contacto con el paciente?

7. ¿Cómo de importante valoras la higiene de manos después del contacto con el paciente?

8. ¿Cuál es tu grado de acuerdo con la siguiente afirmación?: cuando se utilizan guantes puede prescindirse de la higiene de manos

9. ¿Cuál es tu grado de acuerdo con la siguiente afirmación?: llevar anillos o pulseras aumenta la probabilidad de contaminación de las manos

10. ¿Cuál es tu grado de acuerdo con la siguiente afirmación?: la mejor alternativa para la higiene de manos es el lavado con agua y jabón

11. Realizarías más higiene de manos si tus profesores la efectuaran durante las prácticas clínicas

12. ¿Crees que los pacientes y sus familiares deberían preguntar al profesional si ha hecho higiene de manos antes del contacto con aquellos?

Valoración: 0, nada importante; 1, muy poco importante; 2, poco importante; 3, algo importante; 4, bastante importante; 5, muy importante; 6, de total importancia.

ron las siguientes actividades de forma grupal durante seis horas:

- Toma de muestras ambientales.

- Impresión de huellas digitales en placas de agar.

- Supuestos prácticos en los que el participante debía ejecutar correctamente y en el momento adecuado la técnica de higiene de manos.

- Brain-storming sobre cómo mejorar el cumplimiento de la higiene de manos por parte de los profesionales sanitarios.

En el segundo día, se observó el crecimiento bacteriano resultante de la toma de muestras ambienta-
Tabla II. Variables de observación directa sobre higiene de manos.

Zonas contaminadas después del lavado (marcar con una X):

$$
\begin{aligned}
& \text { Palma (_ ) } \\
& \text { Dorso (_ }) \\
& \text { Pulgar (_ }) \\
& \text { Uñas (_ ) } \\
& \text { Pulpejos (_ ) } \\
& \text { Interdigital (_ })
\end{aligned}
$$

Número total de zonas contaminadas:

(se considera 'zona contaminada' aquella que no emite fluorescencia cuando se introducen las manos en la lámpara)

Cumplimiento de los pasos del lavado de higiene de manos por parte de la Organización Mundial de la Salud (sí/no):

1. Cuando se lava las manos aplica suficiente agua y jabón para cubrir toda la superficie de éstas

2. En el proceso de lavado de manos, frota las manos entre sí

3. En el proceso de lavado, frota la palma de la mano derecha contra el dorso de la mano izquierda entrelazando los dedos entre sí, y viceversa

4. En el proceso de lavado, frota los dedos de las manos entre sí con los dedos entrelazados

5. En el proceso de lavado, se frota el dorso de los dedos de una mano contra la palma de la mano opuesta, manteniendo unidos los dedos

6. En el proceso de lavado, rodea el pulgar izquierdo con la palma de la mano derecha frotándola con un movimiento de rotación, y viceversa

7. En el proceso de lavado, se frota la punta de los dedos de la mano derecha contra la palma de la mano izquierda, haciendo un movimiento de rotación, y viceversa

les, así como de las placas de agar donde se habían hecho las impresiones digitales. Además, los participantes expusieron formalmente los resultados de sus trabajos grupales donde proponían estrategias para incrementar el cumplimiento de la higiene de manos. El tiempo de duración fue de tres horas.

\section{Evaluación del taller}

\section{Cuestionario autoadministrado diseñado ad hoc (Tabla I)}

Sirvió para evaluar los conocimientos declarativos básicos y las actitudes hacia la higiene de manos al inicio y al final de la actividad. El cuestionario comprende 12 ítems (del 1 al 10, aspectos declarativos, y del 11 al 12, aspectos actitudinales). Para el análisis se estimó la puntuación media de cada ítem y la 
Tabla III. Puntuación media obtenida para cada uno de los ítems del cuestionario antes del taller (inicial) y después de ése (final) $(n=40)$.

\begin{tabular}{ccccc}
\hline Ítems $^{\mathrm{a}}$ & Media inicial & Media final & $t$ & $p$ \\
\hline Ítem 1 & 5,64 & 5,87 & $-2,160$ & $0,037^{\mathrm{b}}(-0,447 ;-0,140)$ \\
\hline Ítem 2 & 3,82 & 4,92 & $-5,593$ & $0,000^{\mathrm{b}}(-1,500 ;-0,703)$ \\
\hline Ítem 3 & 5,13 & 5,72 & $-4,049$ & $0,000^{\mathrm{b}}(-0,885 ;-0,295)$ \\
\hline Ítem 4 & 3,54 & 3,03 & 1,707 & 0,096 \\
\hline Ítem 5 & 3,67 & 3,03 & 2,262 & $0,029^{\mathrm{b}}(0,070 ; 1,210)$ \\
\hline Ítem 6 & 5,31 & 5,72 & $-1,983$ & $0,050^{\mathrm{b}}(-0,829 ;-0,090)$ \\
\hline Ítem 7 & 5,28 & 5,82 & $-2,683$ & $0,011^{\mathrm{b}}(-0,945 ;-0,132)$ \\
\hline Ítem 8 & 1,13 & 0,51 & 2,309 & $0,026^{\mathrm{b}}(-0,076 ;-1,150)$ \\
\hline Ítem 9 & 5,21 & 5,87 & $-3,757$ & $0,001^{\mathrm{b}}(-1,020 ;-0,307)$ \\
\hline Ítem 10 & 3,62 & 3,59 & 0,066 & 0,948 \\
\hline Ítem 11 & 4,46 & 4,72 & $-0,741$ & 0,463 \\
\hline Ítem 12 & 2,79 & 2,36 & $-1,222$ & 0,229 \\
\hline Media global & 5,64 & 5,87 & $-4,662$ & $0,000^{\mathrm{b}}(-0,580 ;-0,230)$ \\
\hline
\end{tabular}

a Véase la tabla l; b Estadísticamente significativo para $p<0,05$.

global, teniendo en cuenta que el ítem 8 estaba redactado inversamente, y se realizó una comparación de las puntuaciones medias antes y después $(t$ de Student para muestras dependientes). Los registros se llevaron a cabo antes de la charla introductoria (primer día) y justo antes de la entrega de diplomas (segundo día).

\section{Observación directa de la calidad del lavado de manos y de la técnica empleada (Tabla II)}

Se utilizó un preparado de base alcohólica marcado (GlitterBug ${ }^{\circledR}$ Potion) y una lámpara de luz ultravioleta en campo oscuro (Maxi View Box, de GlitterBug ${ }^{\circledR}$ ) para revelar las zonas que no se habían expuesto a la solución (zonas que no emiten fluorescencia al introducir las manos en la lámpara). Dos 'estudiantes-monitores' observaron la técnica de higiene de manos atendiendo a los siguientes pasos, propuestos por la Organización Mundial de la Salud (OMS):

- Suficiente cantidad de solución alcohólica.

- Friccionar palma contra palma.
Tabla IV. Pasos recomendados por la Organización Mundial de la Salud para la higiene de manos antes y después del taller $(n=40)$.

\begin{tabular}{|c|c|c|c|c|}
\hline Pasos a & & No realiza & Sí realiza & $\begin{array}{l}\text { McNemar para } \\
\text { antes y después }\end{array}$ \\
\hline \multirow{2}{*}{ Paso 1} & Antes & 15 & 25 & \multirow{2}{*}{0,000} \\
\hline & Después & 2 & 38 & \\
\hline \multirow{2}{*}{ Paso 2} & Antes & 11 & 29 & \multirow{2}{*}{0,031} \\
\hline & Después & 0 & 40 & \\
\hline \multirow{2}{*}{ Paso 3} & Antes & 25 & 15 & \multirow{2}{*}{0,000} \\
\hline & Después & 1 & 39 & \\
\hline \multirow{2}{*}{ Paso 4} & Antes & 28 & 12 & \multirow{2}{*}{0,000} \\
\hline & Después & 0 & 40 & \\
\hline \multirow{2}{*}{ Paso 5} & Antes & 33 & 7 & \multirow{2}{*}{0,000} \\
\hline & Después & 11 & 29 & \\
\hline \multirow{2}{*}{ Paso 6} & Antes & 34 & 6 & \multirow{2}{*}{0,000} \\
\hline & Después & 0 & 40 & \\
\hline \multirow{2}{*}{ Paso 7} & Antes & 34 & 6 & \multirow{2}{*}{0,000} \\
\hline & Después & 3 & 37 & \\
\hline
\end{tabular}

a Véase la tabla II.

- Friccionar dorso contra palma de la mano contraria y viceversa.

- Friccionar las palmas con los dedos entrelazados.

- Friccionar el dorso de los dedos de una mano con la palma opuesta, agarrándose los dedos.

- Friccionar de forma rotatoria los pulgares.

- Friccionar las puntas de los dedos contra la palma contraria y viceversa.

Posteriormente registraron las regiones de las manos no expuestas (palmas, dorsos, zonas interdigitales, pulpejos, uñas y pulgares) utilizando la tabla II. Cada participante fue evaluado antes de la charla introductoria (primer día) y justo después de las actividades grupales (primer día).

Para el análisis de los resultados se usó el test de McNemar para comparar proporciones apareadas.

\section{Consideraciones éticas}

El estudio se llevó a cabo con la autorización de todos los asistentes y con el consentimiento de poder 
ser utilizado para comunicaciones o artículos científicos. No obstante, los datos recogidos fueron anónimos. Para emparejar los cuestionarios realizados al inicio y al final se pidió a cada uno de los participantes que escribieran un pseudónimo en la parte superior del cuestionario.

\section{Resultados}

Se observó una mejora global significativa de los conocimientos $(p<0,000)$ y unas actitudes más favorables hacia la higiene de manos después de la realización del taller práctico; en 8 de los 12 ítems del cuestionario se detectaron diferencias significativas (Tabla III).

En relación con la técnica de lavado se comprobó que mejoró significativamente el cumplimiento de los pasos propuestos por la OMS para una técnica correcta de higiene de manos con preparados de base alcohólica (Tabla IV). También mejoró la calidad de la higiene de manos. Se observaron diferencias estadísticamente significativas para la media total de zonas contaminadas antes y después del taller: 11,40 frente a 3,11, respectivamente $(t=9,278$; $p<0,000)$. Igualmente, las diferencias fueron significativas para el porcentaje de alumnos con zonas no expuestas a la solución alcohólica, que disminuyó en todas las regiones anatómicas de las manos (Tabla V), excepto en los pulpejos de los dedos.

\section{Discusión}

Los resultados expuestos parecen indicar que el taller práctico impartido y liderado por los propios estudiantes, con asistencia tutelada, mejora la técnica, los conocimientos y las actitudes del colectivo estudiantil en relación con la higiene de manos.

El liderazgo por parte de los estudiantes es el gran valor y la aportación diferencial con respecto a otras estrategias educativas llevadas a cabo con personal sanitario en formación y, sin duda, es uno de los valores fundamentales del trabajo. Son muy pocos los estudios en los que se brinda el liderazgo de la formación al estudiante [9], pero a estas actividades se les atribuye un gran valor educativo debido a la adquisición de un compromiso activo que incide sobre la conducta posterior. Este tipo de actividades formativas debería plantearse como una estrategia complementaria para concienciar a los profesionales del futuro sobre la importancia de la higiene de manos en la asistencia sanitaria, además de potenciar habilidades en los 'estudiantes-moni-
Tabla V. Calidad de la higiene de manos con solución alcohólica (luminiscencia observada con lámpara ultravioleta) $(n=40)$.

\begin{tabular}{|c|c|c|c|c|}
\hline & & $\begin{array}{l}\text { Persisten zonas } \\
\text { no marcadas }\end{array}$ & $\begin{array}{c}\text { Correctamente } \\
\text { expuesta }\end{array}$ & $\begin{array}{l}\text { McNemar para } \\
\text { antes y después }\end{array}$ \\
\hline \multirow{2}{*}{ Palmas } & Antes & 29 & 11 & \multirow{2}{*}{0,000} \\
\hline & Después & 5 & 35 & \\
\hline \multirow{2}{*}{ Dorsos } & Antes & 33 & 7 & \multirow{2}{*}{0,000} \\
\hline & Después & 15 & 25 & \\
\hline \multirow{2}{*}{ Pulgares } & Antes & 32 & 8 & \multirow{2}{*}{0,000} \\
\hline & Después & 14 & 26 & \\
\hline \multirow{2}{*}{ Uñas } & Antes & 32 & 8 & \multirow{2}{*}{0,000} \\
\hline & Después & 10 & 30 & \\
\hline \multirow{2}{*}{ Pulpejos } & Antes & 30 & 5 & \multirow{2}{*}{1,000} \\
\hline & Después & 30 & 5 & \\
\hline \multirow{2}{*}{$\begin{array}{l}\text { Regiones } \\
\text { interdigitales }\end{array}$} & Antes & 26 & 14 & \multirow{2}{*}{0,000} \\
\hline & Después & 7 & 33 & \\
\hline
\end{tabular}

tores', favorecer el asociacionismo y la participación estudiantil dentro de las labores formativas extracurriculares del centro y, por último, generar competencias relevantes para el profesional sanitario en el marco de los nuevos planes de estudio, como la comunicación, el liderazgo, la gestión, etc., competencias generales que todo profesional debe adquirir a su paso por la universidad.

En el taller se ha utilizado como guía el trabajo realizado por otros autores [10] sobre el entrenamiento de estudiantes de medicina en el uso de soluciones alcohólicas mediante lámparas ultravioleta. Al igual que estos autores, encontramos que es una técnica de retroalimentación que mejora el aprendizaje de la técnica en los estudiantes.

No obstante, los resultados obtenidos no demuestran que esta metodología docente sea mejor que otras donde el profesorado tiene un papel principal y directivo en todo el proceso, ya que no tenemos datos que lo avalen. Sólo entendemos que las actividades lideradas por estudiantes para estudiantes, con apoyo y tutela académica, son una estrategia educativa válida que se necesita reforzar y con la que se obtienen resultados satisfactorios.

Si bien pudiera pensarse que diez horas es un tiempo excesivo para la duración de un taller de este tipo, hay que remarcar que es una actividad impar- 
tida por estudiantes durante casi su totalidad y que éstos carecen de experiencia docente. Además, el taller presenta diversos procesos como toma de muestras y recogida de datos, así como actividades en grupo, que incrementan su duración. Con todo lo expuesto, consideramos que la duración es la apropiada y que podría disminuir en posteriores ediciones si los monitores fueran los mismos.

En la tabla III se observa cómo se incrementa la importancia concedida a la higiene de manos en el contexto sanitario y cómo la realización del taller implica una visión más crítica del cumplimiento de higiene de manos por el personal sanitario (ítems 4 y 5). El ítem 11 muestra el importante papel del aprendizaje vicario en la formación y es consistente con otros trabajos [11,12], al igual que el ítem 12, según el cual la implicación de agentes externos a la sanidad (familiares) no se percibe como una estrategia positiva. Destaca la escasa mejora percibida sobre la utilidad de los preparados de base alcohólica para la higiene de manos, posiblemente indicativo de resistencias al cambio fruto del propio aprendizaje vicario [11].

Al analizar la tabla IV, destaca que los estudiantes han aprendido correctamente la técnica de higiene de manos a través de los pasos propuestos. Se aprecia en la tabla $\mathrm{V}$ que si bien los estudiantes mejoran, existen todavía deficiencias en la calidad del lavado.

En conclusión, el taller impartido mejoró las actitudes, los conocimientos y las habilidades en cuanto a la técnica de higiene de manos entre los participantes. No obstante, existen algunas limitaciones en el trabajo que se subsanarán en ediciones futuras:

- Necesidad de validar el cuestionario para evaluar conocimientos y actitudes.

- Mayor número de participantes o sesiones del taller.

- Hacerlo extensivo a otras facultades y universidades.
- Comparar esta metodología docente con un curso impartido sólo por profesores y analizar posibles diferencias en cuanto al aprendizaje de los participantes.

\section{Bibliografía}

1. Klevens RM, Edwards JR, Richards CL, Horan TC, Gaynes RP, Pollock DA, et al. Estimating health care-associated infections and deaths in U.S. hospitals, 2002. Public Health Rep 2007; 122: $160-6$.

2. Helder OK, Brug J, Looman CWN, Van Goudoever JB, Kornelisse RF. The impact of an education program on hand hygiene compliance and nosocomial infection incidence in an urban Neonatal Intensive Care Unit: an intervention study with before and after comparison. Int J Nurs Stud 2010; 47: 1245-52.

3. Conly JM, Hill S, Ross J, Lertzman J, Louie TJ. Handwashing practices in an intensive care unit: the effects of an educational program and its relationship to infection rates. Am J Infect Control 1989; 17: 330-9.

4. Pittet D. Improving compliance with hand hygiene in hospitals. Infect Control Hosp Epidemiol 2000; 21: 381-6.

5. Haas JP, Larson EL. Measurement of compliance with hand hygiene. J Hosp Infect 2007; 66: 6-14.

6. Henderson D, Carson-Stevens A, Bohnen J, Gutnik L, Hafiz S, Mills S. Check a box. Save a life: how student leadership is shaking up health care and driving a revolution in patient safety. J Patient Saf 2010; 6: 43-7.

7. Hoffman SJ, Rosenfield D, Gilbert JHV, Oandasan IF. Student leadership in interprofessional education: benefits, challenges and implications for educators, researchers and policymakers. Med Educ 2008; 42: 654-61.

8. Duvivier R, Rodríguez-Muñoz D. La participación de los estudiantes en la educación médica. Educ Med 2010; 13: 223-7.

9. Bicket M, Misra S, Wright S, Shochet R. Medical student engagement and leadership within a new learning community. BMC Med Educ 2010; 10: 20.

10. Hautemeniére A, Diguio N, Dayal MC, Hunter PR, Hartemann P. Short-term assessment of training of medical students in the use of alcohol-based hand rub using fluorescent-labeled hand rub and skin hydration measurements. Am J Infect Control 2009; 37: 338-40.

11. González-Cabrera J, Fernández-Prada M, Martínez-Bellón MD, Fernández-Crehuet M, Guillén J, Bueno-Cavanillas A. Construcción y validación de un cuestionario para medir conductas, conocimientos y actitudes sobre la higiene de las manos en personal sanitario. Rev Esp Salud Publica 2010; 84: 827-41.

12. Snow M, White G, Alder S, Stanford J. Mentor's hand hygiene practices influence student's hand hygiene rates. Am J Infect Control 2006; 34: 18-24. 